\title{
The Role of Artificial Intelligence in The Decision-Making Process at The Ministry of Interior in The United Arab Emirates
}

\author{
Shaikha Saif Almansoori, Ali Nasser Al-Tahitah* \\ Faculty of Leadership and Management, Islamic Science University of Malaysia (USIM), Negeri Sembilan, \\ Malaysia \\ *alinasser@usim.edu.my
}

\begin{abstract}
KEYWORDS: $\quad$ Artificial Intelligence, Decision-Making Process, The Ministry of Interior.
\end{abstract}
\begin{abstract}
:
This research paper is part of an extensive study conducted by the researcher on the UAE strategy for artificial intelligence, and its role in changing the mechanism of government services, leading to a complete data analysis by 2031, according to the latest techniques and tools of artificial intelligence and their application in various fields of work efficiently. The problems of smart devices lie in the bubble filter phenomenon, which causes a lack of elasticity, increased systemic fragility, which can have serious and unexpected systemic effects. The paper objectives were; to discuss the decisionmaking process and the employment of artificial intelligence in the United Arab Emirates. Through the Descriptive Analytical method, the topic was presented in two sections. The discussions showed that the UAE's strategy for artificial intelligence targets several vital sectors in the country, including the transportation sector, by reducing accidents and operational costs. The health sector by reducing the proportion of chronic and dangerous diseases. The space sector conducts accurate experiments and reduces costly errors. Energy sector through utility management and smart consumption. The water sector by conducting accurate analysis and studies to save resources. The technology sector by raising the rate of production and helping in public spending, the education sector by reducing costs and increasing the desire to learn. The environment sector by increasing the proportion of afforestation and planting suitable plants. Traffic Sector Develop preventive mechanisms such as predicting accidents and traffic congestion, and setting more effective traffic policies. With the presence of the infrastructure and knowledge in the successful recruitment of artificial intelligence in the Ministry of Interior in the United Arab Emirates.
\end{abstract}

\section{REFERENCES:}

Bates, John, (2015). Post Flash. Traders Magazine Online News, archived from the original on January 25, 2018, retrieved April 25, 2014

Creswell, JW (2012) Education research: planning, implementation, and evaluation Quantitative and Qualitative Research Fourth Edition University of Nebraska-Lincoln.

Dorian Combe (2018) The roles of artificial intelligence and decision-making Mankind: Toward Enhanced Humans? Knowledge-intensive companies focus, Department of Business Administration, UMEA Swedish Master's Program in Busines Development

Dorian Combe (2018) The roles of artificial intelligence and decision-making Mankind: Toward Enhanced Humans Knowledge-intensive companies focus, Department of Business Administration, UMEA Swedish Master's Program in Business Development.

Eli Pariser, (2012). The Filter Bubble: How the New Personalized Web Is Changing What We Read and How We Think Paperback. Penguin Books, ISBN-10: 0241954525

Jo Young Lee (2009) on the effect of computer-mediated Communication (CMC) interaction on vocabulary acquisition the second language; A comparative study of CMC interaction and face-to-face interaction at Iowa Ames State University, Iowa. 
Li, B. H., Hou, B. C., Yu, W. T., Lu, X. B., \& Yang, C. W. (2017). Applications of artificial intelligence in intelligent manufacturing: a review. Frontiers of Information Technology \& Electronic Engineering, 18(1), $86-96$.

Nuti, Giuseppe, Mahnoosh Mirghaemi, Philip Treleaven, and Chaiyakorn Yingsaeree. "Algorithmic Trading," Computer, Vol. 44, No. 11, 2011, pp. 61-69.

Osoba, Osonde A., and William Welser (2017), An Intelligence in Our Image: The Risks of Bias and Errors in Artificial Intelligence, Santa Monica, Calif.: RAND Corporation, RR-1744-RC, 2017. 
دور الذكاء الاصطناعي في عملية اتخاذ القرارات في وزارة الداخلية بدولة الإمارات العربية المتحدة

\author{
شيخة سيف المنصوري، د. علي ناصر الطحيطاح

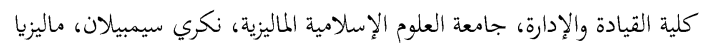 \\ * alinasser@usim.edu.my
}

الكلمات المفناحية | الذكاء الاصطناعي، عملية اتخاذ القرارات، وزارة الداخلية.

Crossref doi $\quad$ https://doi.org/10.51345/.v32i3.451.g237

\title{
ملخص البحث:
}

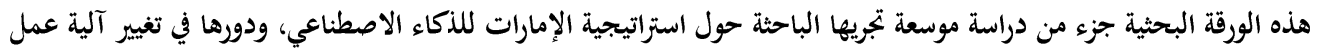

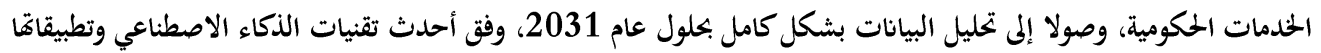

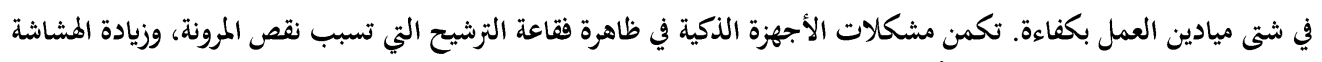

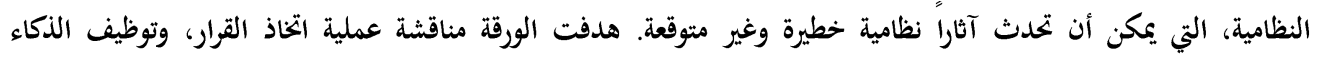

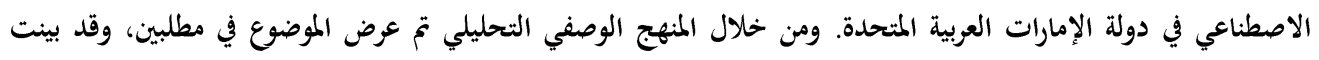

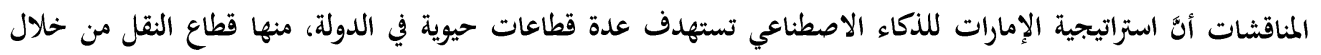

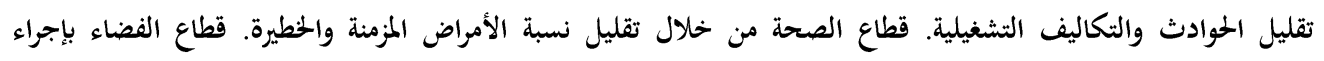

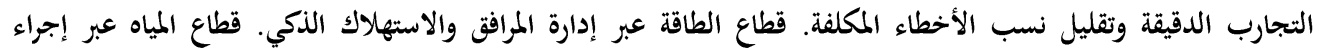

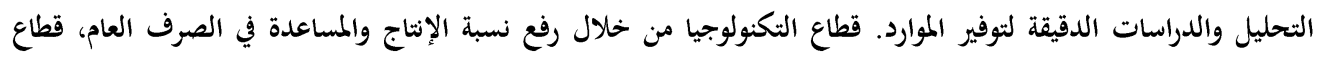

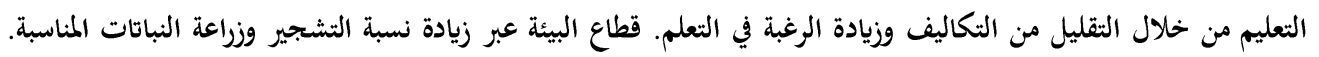

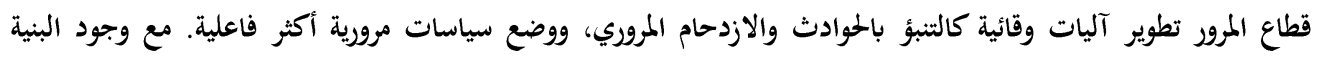

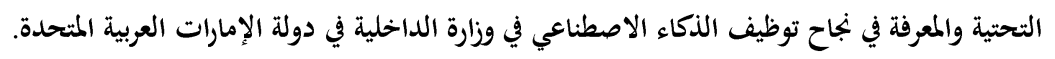

المقدمة:

يعرَّف الذكاء الاصطناعي على أنه النطاق الواسع الذي يشمل كافة العمليات من النبوغ والابتكار والتحكم

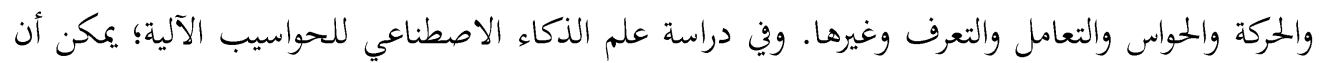

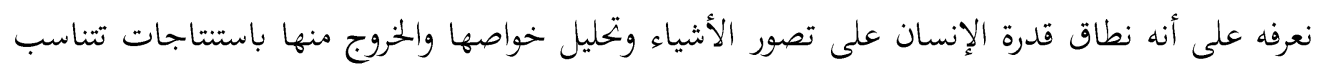
مع الآلة، فهي إذا القدرة على تطوير نموذج ذهني تتمثل فيه عناصر واستخلاص ونتائج وعلاقات البشر بما لهات 
يتحد مع الآلة ويهقق النتائج الإيجابية لمستقبل الججتمعات بحيث يتفاعل مع المواقف والتعامل معها بشكل آلي دون الحاجة إلى البشر مستقبلاًأ(1). كما يعرف الذكاء الاصطناعي على أنه قدرة الآلة على محاكاة العقل البشري وطريقة عمله، مثل قدرته على الفيتل

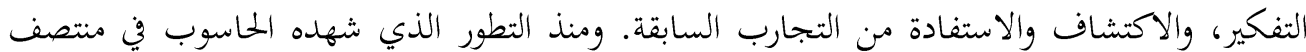

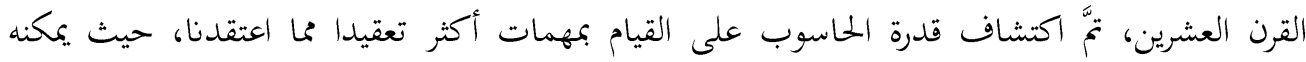
اكتشاف الإثباتات للنظريات الرياضية المعقدة بالإضافة لقدرته على لعب الشطرنج بمهارة كبيرة، ومع ذلك الكابك

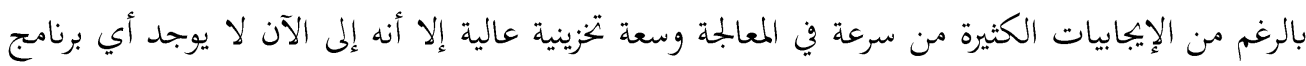

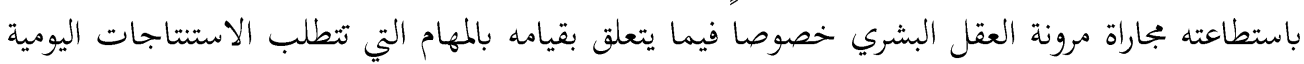
التلقائية لما يتم التعرض له"(2). وتقدم سياسة الذكاء الاصطناعي في الإمارات العربية المتحدة ملخصاً من فبراير 2020 لبعض المبادرات

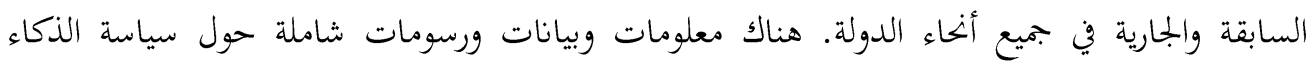

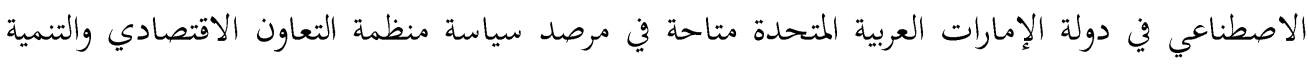

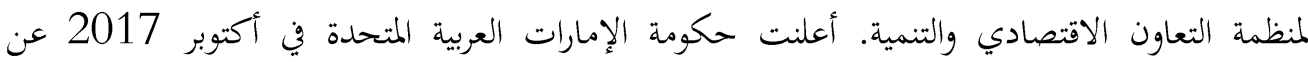

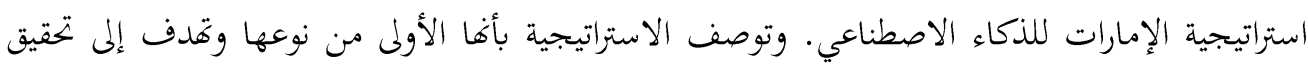

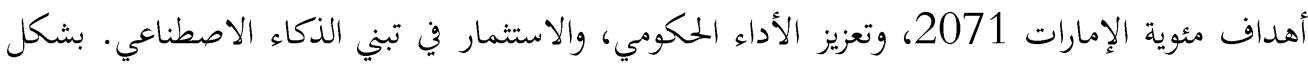

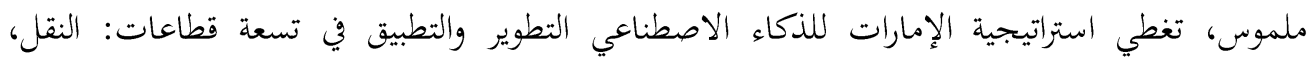
والصحة، والفضاء، والطاقة المتجددة، والمياه، والتكنولوجيا، والتعليم، والبيئة، وحركة المرور.

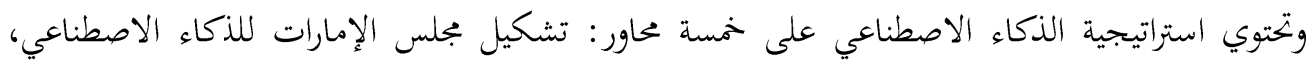

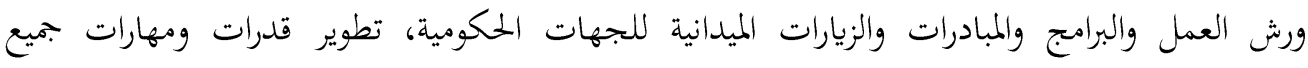

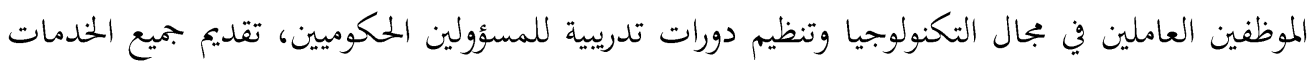

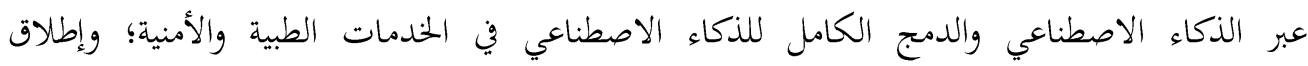

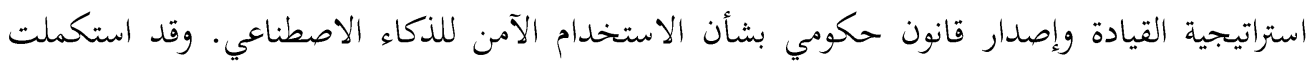

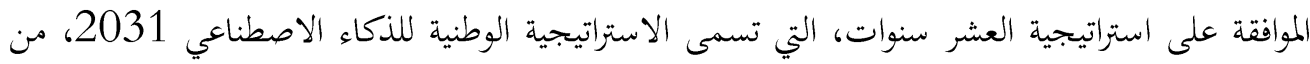
قبل مجلس الوزراء في أبريل 2019. 


\section{مشكلة الدراسة:}

لا شك أنَّ توفير البيانات الصحيحة من شأنه أن يعين قيادة المنظمة في صنع واتخاذ القرارات الصحيحة.

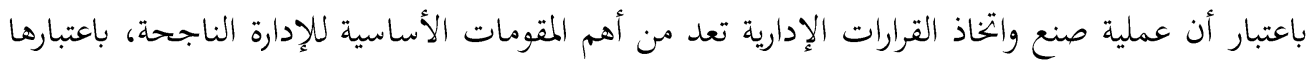

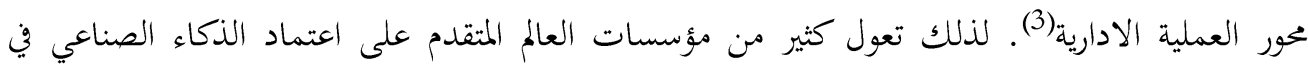

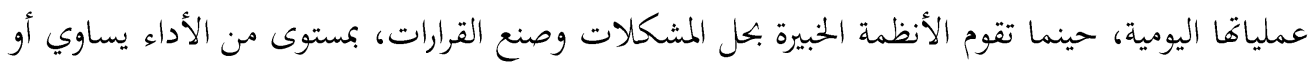

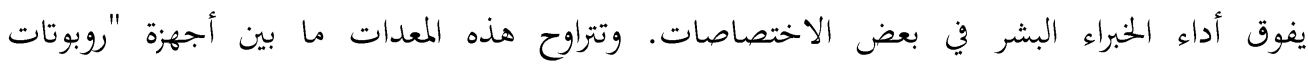

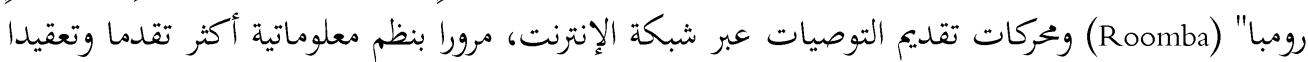
مثل واتسون (Watson) من شركة "آي بي إم" (IBM). وقد أصبحت تلك الأجهزة الذكية جزء لا يتجزأ من إجراءات المنتظمات المعتمدة في صنع القرارات الإدارية. إلآلا إن استخدام هذه التقنيات رافقته تحديات

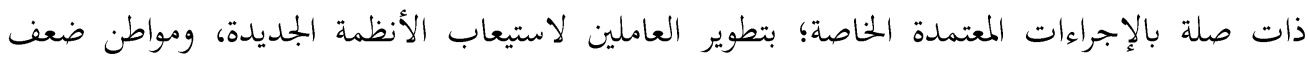
الإنسان في هذا البجال، وكيف يمكن المستخدمين والأفراد تحديد الأخطاء التي تشوب النتائج المنطقية لأداء الأجهزة وكيفية حلها، والمنظمات الأكثر جهوزية وحاجة للذكاء الاصطناعي.

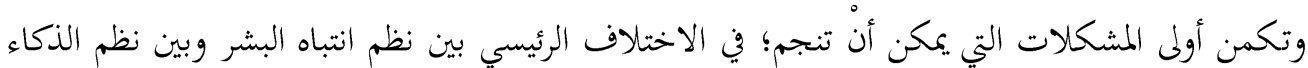
الاصطناعي لدى معالجة البيانات. إذ تتمتع أدمغتنا البشرية حاليا بمرونة محدودة، وهذا يمكيّن أطر انتبناه

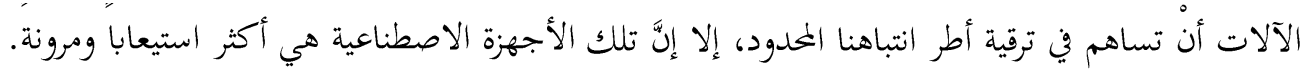

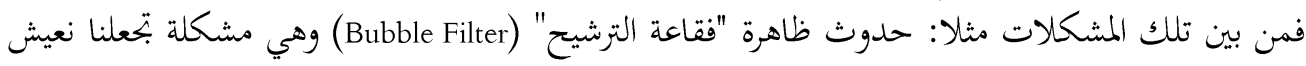
في بيئة متجانسة ومتوافقة بشكل كبير مع من نكون حسبما تمليه علينا الأجهزة، دون التعرض لأفكار

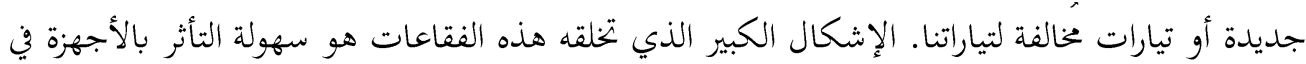
توجيه الرأي العام(4). حيث إننا في الغالب نسعى إلى تجنب حمل المعلومات الزائدة. ففي إطار صنع القرارات نقوم بتفويض مهام معالجة المعلومات الفرعية للأجهزة؛ لنتمكن نهن من التركيز على القرارئ التهات الرئيسية. ولربما يتحسن النطاق المتوفر حاليا مع بروز ابتكارات جديدة في تكنولوجيا المعلومات. النوع الاخر من التحديات، هو التسبب في إحدى نتيجتين غير مرغوب فيهما، الأولى: تناقص المرونة بسبب محدودية المعلومات المتوفرة لدينا، حول كيفية تأثير الأتمتة في حياتنا. ونعني (resilience diminished) بذلك إمكانية الخفاض المرونة النظامية، أما الثانية: فهي زيادة الهشاشة النظامية، عبر الاعتماد بصورة أكبر على الأدوات الاصطناعية. ويمكن لتقلص المرونة أن يتخذ نظرة مفرطة الثقة للأدوات الاصطناعية أو نظرة 
غير ناقدة بما يناسبها. وقد بدأت تتضح هذه الثقة غير المبررة في تعاملنا مع الأدوات الاصطناعية البدائية التي نستخدمها حاليا. وهذا خير دليل على ميل البشر نهو التحيز للأتمتة.

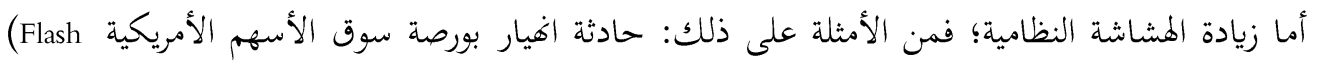

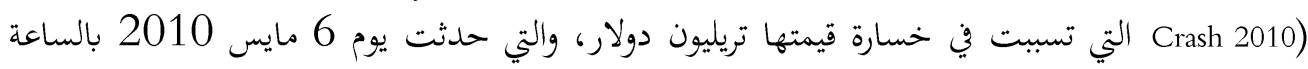
2:32 مساء واستمرت لمدة 36 دقيقة (Bates, John, 2015). كانت تلك الحادثة مثالا على ظهور أشكال جديدة من الهشاشة النظامية، جراء استعمال الأدوات الاصطناعية (Nuti, et. al, 2011). وغالبا ما يتخذ هذا التناقص في المرونة شكل نظرة مفرطة الثقة بالأدوات الاصطناعية، أو نظرة غير نقدية لما تستحقه. وقد أشارت دراسات عدة، منها: القربي (2012)، الخزيم (2012)، القحطاني (2013) إلى تحديات

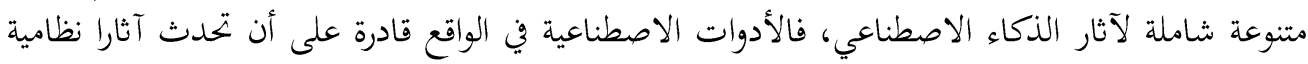

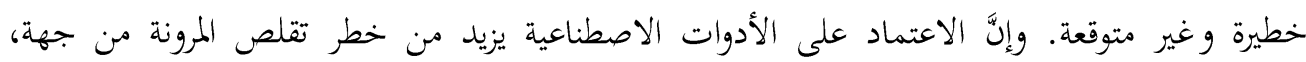
والتسبب بفوضى اقتصادية واجتماعية سريعة غير مسبوقة. وذكرت قمورة، (2018) إنَّ بعض العلماء

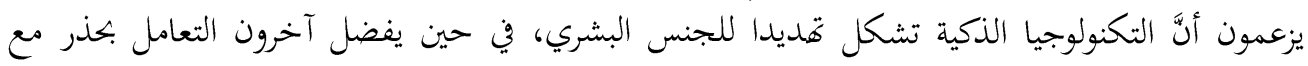
الأدوات الذكية.

لذلك يمكن القول بأنه، رغم التهافت الإعلامي على الذكاء الاصطناعي، ووصوله إلى مستويات عالية من القدرات فوق البشرية، وعن خوارزميات تزم الأطباء في التشخيص، وإنَّ الحاسوب قادر على على حل جميع

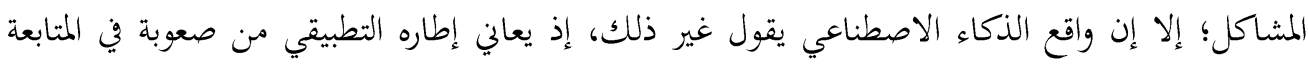

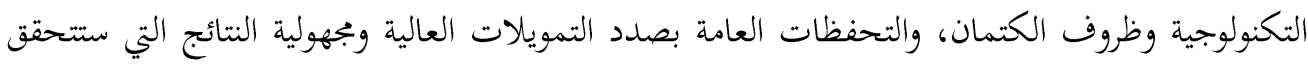
(قمورة، 2018). ويثير الذكاء الاصطناعي قضايا جديدة للمنظمات فيما يتعلق بمسؤوليته وقبوله من قبل

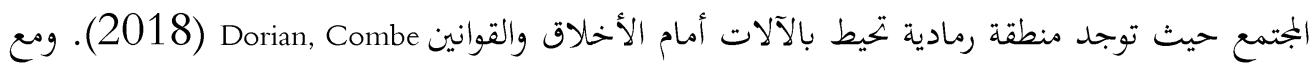
تلك التحديات يستمر دور الذكاء الاصطناعي في السير قدما، في جميع ميادين حياتنا في قرن الألفية الثالثة

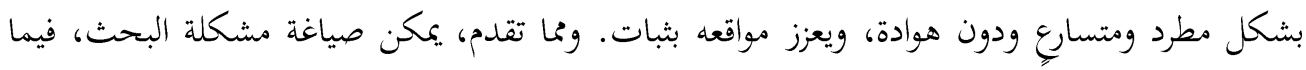
يأتي: مناقشة دور الذكاء الاصطناعي في عملية اتخاذ القرارات في وزارة الداخلية بدولة الإمارات العربية

\section{أهداف الدراسة:}

أ. تحليل خطوات عملية اتخاذ القرار.

ب. مناقشة دور الذكاء الاصطناعي في الاستشراف في وزارة الداخلية بدولة الإمارات العربية المتحدة. 


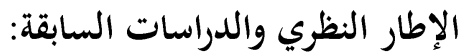

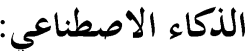

يعرف المهيري (2019) الذكاء الاصطناعي على أنه: الوسيلة التي يستخدمها الإنسان لحل المشكلات

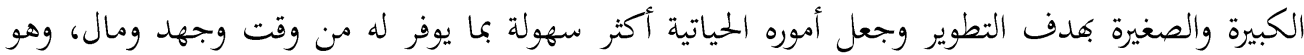
القادر على جعل الآلة تعمل بخصائص الإنسان وتفكر بطريقته بجيث تكون قادرة على تلبية المتطلبات بطريقة ذكية تساهم في تحقيق الأهداف المرجوة منها بالإضافة إلى قدرتا على قراءة الأشياء ومقارنتها

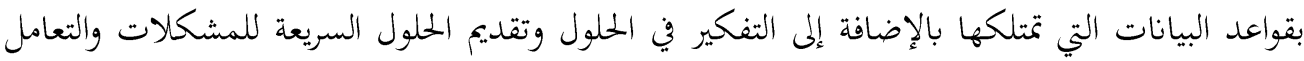

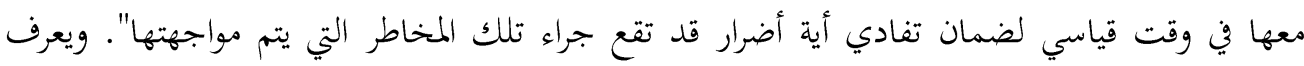

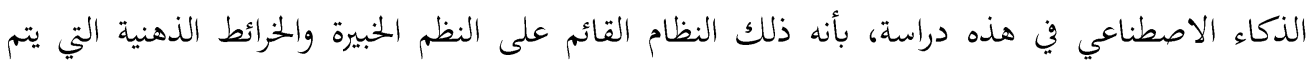

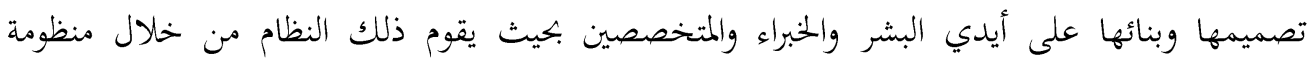

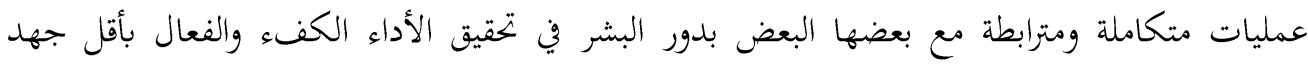

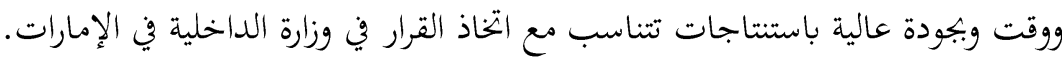

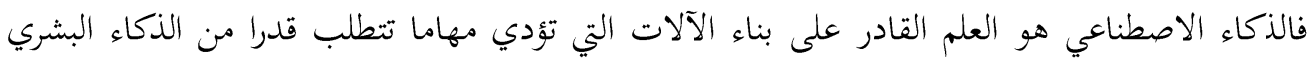

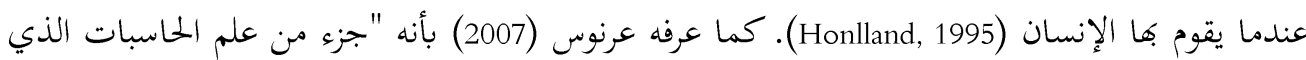

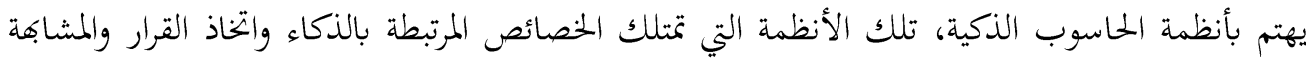

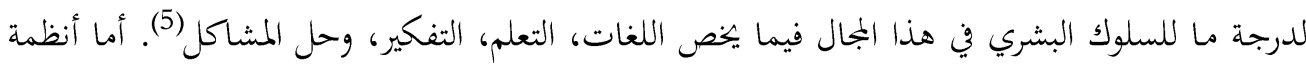
الذكاء الاصطناعي فهي حقل واسع يهتم بتطوير الحاسبات لتقوم بتنفيذ المهام التي تتطلب ذكاء إنسانيا.

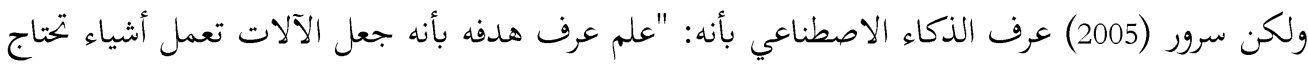

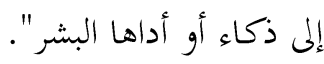

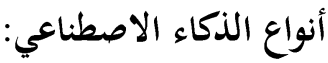

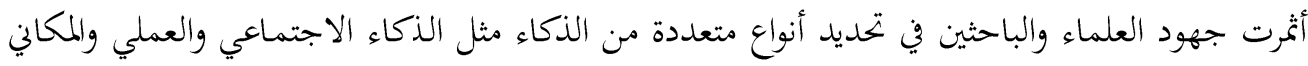

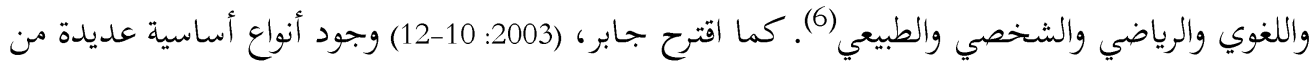

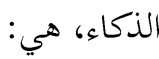


أولا. الذكاء اللغوي (Linguistic Intelligence): يعبر الذكاء اللغوي عن قدة الشخص على استخدام الكلمات بفاعلية. ويعتقد البعض أنه وفي حال كان الفرد يتقن أكثر من لغة بطلاقة، فإنه يتمتع بذكاء لغوي ملحوظ. لكن إتقان عدة لغات ليس الأمر الوحيد الذي يجعل من الشخص ذكيا لغويا، فربما يعرف لغة واحدة فقط، لكنه مع ذلك يتمتع بقدرات عالية يي استخدام هذه اللغة بالذات. بمعنى آخر، فالذكاء اللغوي يعبر عن قدرة الفرد على استخدام الكلمات بطريقة مميزة توضح ما يعنيه. ثانيا. الذكاء المنطقي الرياضي (Logical Mathematical Intelligence): غالبا ما يقاس ذكاء الشخص بناء على قدراته المنطقية، أو مهاراته في حل المسائل الرياضية. وهذا أحد أنواع الذكاء وأشهرها، لكنه ليس الوحيد بالطبع. يعبر الذكاء المنطقي عن منطق استقرائي واستناجي لدى الشخص بالإضافة إلى مهارات حسابية مميزة. باستطاعة الشخص الذي يمتلك هذا النوع من الذكاء التفكير بشكل نظري. حيث لا يجد صعوبة في التعرف على الأنماط المختلفة وتحديد العلاقات بينها، وهو يتمتع بترتيب وتسلسل لا مثيل له في التفكير ثالثا. الذكاء البصري المكاني (Visual Spatial Intelligence): عند الحديث عن القدرة على التفكير بطريقة ثلاثية الأبعاد، فذلك يتضمن؛ التصور العقلي أو قدرة الشخص على أن يرسم في مخيلته صورة تمثل العالم المادي. سواء كانت تلك ذكرى من الماضي أو بتربة حقيقية. فهذا الشخص يستطيع أن يرى "في عقله" هذا الشيء بوضوح تام حتى لو لم يكن موجودا أمامه، المنطق المكاني أو القدرة على التفكير بالأشياء بأبعادها الثلاثية، حتى على الرغم من امتلاك معلومات محدودة عنها، ويتضمن ذلك أيضا القدرة على رسم فكرة عامة واضحة بناء على معلومات محددة. معالجة الصور أو القدرة على رؤية صورة معينة وتخيل صورها بعد معالجتها أو إجراء تعديلات عليها. يواجه البعض مشكلة في تصور النتيجة النهائية لصورة أو لوحة معينة في حال تم التعديل عليها، بينما يستطيع البعض الآخر وبكل سهولة تخيل الشكل النهائي لها. رابعاً. الذكاء الشخصي (Interpersonal Intelligence): ترتبط المشاعر ارتباطًا وثيقا بالذكاء أيضا، حتى أن هناك ما يعرف بالذكاء العاطفي الذي يشار له اختصارا بـ .EQ ويعبر الذكاء الشخصي أو العاطفي عن قدرة الشخص على الإحساس بمشاعر الآخرين وقراءة دوافعهم. حيث يعرف عن الأشخاص الذين يتمتعون هذا النوع من الذكاء بأفم يمتلكون مهارات تواصل شفوية وغير شفوية عالية. فالشخص يمتلك هذا النوع من الذكاء إن كان قادرا" على تمييز الاختلافات أو الفروقات بين الأشخاص، وتحديد شخص محدد في حشد كبير. يملك دائرة اجتماعية واسعة تضم العديد من الأصدقاء ويستمتع بقضاء الوقت مع الآخرين. يتمتع بحساسية عالية للمزاجية وطباع الآخرين. يمتلك القدرة على النظر لموضوع معين من وجهات نظر 
مختلفة بدلا" من التمسك بوجهة نظره. بإمكان الأشخاص الذين يمتلكون ذكاء عاطفيا العمل في مجالات

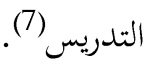

\section{خصائص الذكاء الاصطناعي:}

يتمتع الذكاء الاصطناعي بالعديد من الخصائص والمميزات والقدرات، وقد ذكر النجار، (2010) بعضها منها؛ القدرة على التعامل مع الحالات الصعبة والمعقدة، القدرة على التعامل مع المواقف الغامضة مع غياب المعلومة، القدرة على تمييز الأهمية النسبية لعناصر الحالات المعروضة، القدرة على التصور والإبداع وفهم الأمور المرئية وإدراكها، القدرة على تقديم المعلومة لإسناد القرارات الإدارية. وهناك بجموعة أخرى من العناصر التي يتميز بها الذكاء الاصطناعي؛ نذكر منها: إن برامج الذكاء الاصطناعي على عكس البرامج الإحصائية تحتوي على أسلوب لتمثيل المعلومات إذ تستخدم هيكلة خاصة لوصف المعرفة، وهذه الميكلة تتضمن الحقائق (Facts) والعلاقات بين هذه الحقائق Relationship والقواعد التي تربط هذه العلاقات Rules، وبجموعة الهياكل المعرفية تكون فيما بينها قاعدة المعرفة Knowledge Base وتتسم برجيات الذكاء الاصطناعي عموما "بعدم وجود حل خوارزي معروف للمشاكل التي تتناوها، لذلك لابد من اللجوء إلى الاجتهاد ويتمثل الاجتهاد، في اختيار طرق الحل التي تبدو ملائمة مع الإبقاء على فرصة التغيير إلى طريقة أخرى في حال عدم توصل الطريقة الأولى إلى الحل المنشود في الوقت

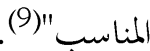

أحد معايير السلوك المتسم بالذكاء هو القدرة على التعلم من الأخطاء وهو ما يؤدي إلى تحسين الأداء نتيجة الاستفادة من الأخطاء السابقة، ولو طبقنا هذا المعيار بحذافيره لما وجدنا سوى عدد قليل من البشر يمكن أن يعدوا أذكياء، وترتبط ملكة تعلم الإنسان من الأخطاء بقدرته على الوصول من الجزئيات إلى العموميات(10)

\section{الدراسات السابقة.}

أجرى (2018) في جامعة Dorian Combe, السويدية، دراسة حول أدوار الذكاء الاصطناعي واتخاذ القرارات البشرية. كان التركيز على الشركات القائمة لتوفير فهم أفضل لدور البشر والذكاء الاصطناعي في عملية صنع القرار التنظيمي. ركز البحث على الثركات القائمة على المعرفة الغزيرة. سؤال البحث الرئيسي للدراسة هو: كيف يمكن للذكاء الاصطناعي إعادة تصميم وتطوير عملية صنع القرار 
التنظيمي داخل الشركات القائمة على المعرفة الغزيرة؟ وتم صياغة ثلاثة أسئلة فرعية: حول أدوار البشر والذكاء الاصطناعي في عملية صنع القرار، ودور التصميم التنظيمي في دعم عملية صنع القرار من خلال استخدام الذكاء الاصطناعي، كيف يساعد الذكاء الاصطناعي في التغلب على التحديات التي يوجهها صناع القرار؟ وما التحديات الجديدة التي تنشأ من استخدام الذكاء الاصطناعي في عملية صنع القرار؟ اعتمدت دراسة نوعية، مثل مجتمع الدراسة شركتان كبيرتان لتكنولوجيا المعلومات، وشكتان عقاريتان

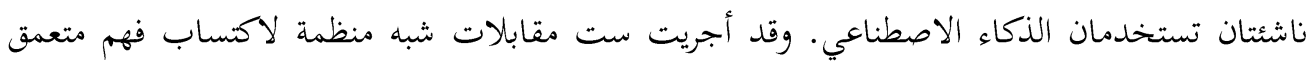
لأدوار البشر والذكاء الاصطناعي في عملية صنع القرار داخل الشركات كثيفة المعرفة. سأَطت الأطروحة الضوء على الفهم العميق للذكاء الاصطناعي، وتكامله في عملية صنع القرار التنظيمي للشركات القائمة على المعرفة الغزيرة، وتمكين البشر من زيادة قدراقم واتحاذ قرارات أكثر ذكاء. وظهر أن الذكاء الاصطناعي يستخدم كدعم لاتخاذ القرار بدلا من صناعة قرار مستقل، وأن المنظمات تتبنى تصميمات أكثر سلاسة وتعاونا لتحقيق أقصى استفادة منها في عملية اتخاذ القرار. الذكاء الاصطناعي هو هوري أداة فعالة للتعامل مع المواقف المعقدة، في حين تبدو القدرات البشرية أكثر أهمية في حالات عدم اليقين والغموض. يثير الذكاء الاصطناعي أيضا قضايا جديدة للمنظمات فيما يتعلق بمسؤوليته وقبوله من قبل المجتمع، حيث توجد منطقة رمادية تحيط بالآلات أمام الأخلاق والقوانين. دراسة مازورك كاترين (2019) في جامعة "بومبيو فابرا"، برشلونة، إسبانيا، حول الإنسان مقابل الذكاء الاصطناعي دراسة سلوكية للمستهلك حول أخذ المشورة بين الجيل (Y) وعلى الرغم من أن الخوارزميات

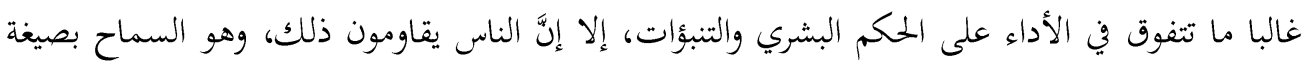

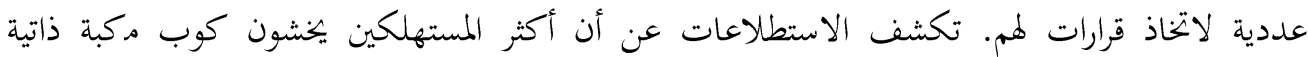
القيادة، ركذلك يفضلون الأطباء البشر على الخوارزميات في السياق الطبي، على الرغم من الأدلة على أن الخوارزميات قد تقدم تشخيصا أكثر دقة (Cremer et al 2019). وعلى عكس هذه الفكرة، أظهرت نتائج الدراسة؛ أن الناس يفضلون نصيحة حاسوبية بدلاً من النصائح البشرية عند إجراء تقديرات رقمية حول التحفيز البصري (التجربة 1 أ)، تنبؤات حول شعبية الأغاني

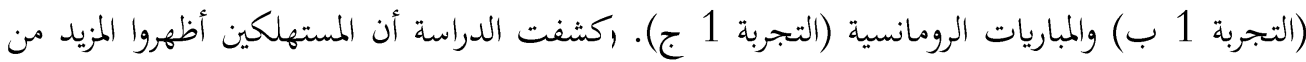
النفور التحاه الخوارزميات عند الاختيار بين التقدير الخوارزمي وتقديراقم الخاصة (مقابل مستشار خارجي). وبالمثل، أظهر المشاركون المزيد من التسامح تجاه اتخاذ القرار الخاطئ الذي يصدر عن إنسان وليس من 
الصيغة. ومن ثم، عند دراسة النطاق الذي يفضل المستهلكون فيه الحوارزمية على النصائح البشرية، من الأهمية بمكان مراعاة التحيزات المعرفية التي تؤثر على عملية صنع القرار.

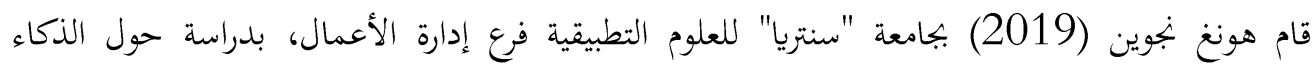
الاصطناعي وأثره على القوة العاملة، وتوضيح وظيفته، ركان الهدف من الأطروحة هو التركيز على الذكاء

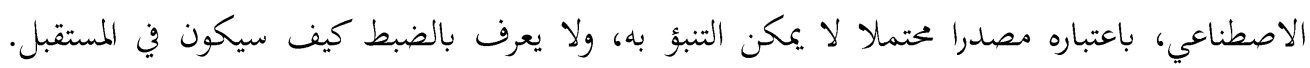

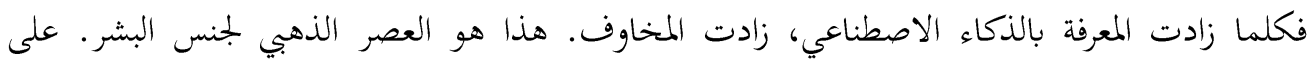

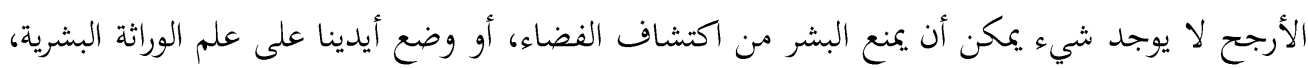

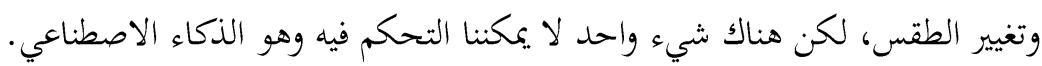
الهدف من هذه الرسالة هو إيجاد حل للناس فيما يتعلق بما سيفعله الطلاب المتخرجين في السنوات القليلة القادمة؟ السؤال ليس شيئا جديدا، ليس لأي طالب ولكن في إلى في الوقت الحالي، سيحتاج الطلاب إلى معرفة بجال جديد تماما لتكنولوجيا المعلومات للإجابة على هذا السؤال. يمنح وجود الذكاء الاصطناعي للبشرية علامة استفهام كبيرة، حتى أن العلوم العليا في العالم الآن لا تستطيع أن تفهم تماما ماهيته وطريقة عمله وإمكانياته. ومع ذلك، ليس هناك شك في قوة الذكاء الاصطناعي وأنه سيصبح أداة تشكل ملامح العالم في السنوات القليلة القادمة. قام راكيل اينارسدوتير (2019) قسم العلوم الاجتماعية بجامعة آيسلاندا، بدراسة حول الذكاء الاصطناعي والإدارة: هل سيحل الذكاء الاصطناعي محل مديري المستوى المتوسط؟ وكان الهدف من تلك إنك الأطروحة هو معالجة فجوة في الأدبيات من خلال إجراء بحث منهجي لمراجعة الأدبيات حول المعرفة الحالية للذكاء الاصطناعي في سياق الإدارة المستقبلية، مع التركيز بشكل خاص على على النتائج المتعلقة بمديري

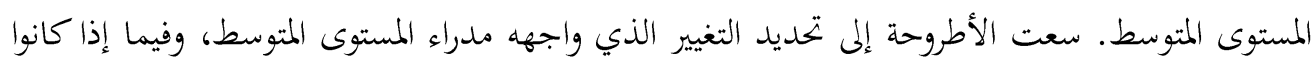
سيكونون زائدين عن الحاجة في المستقبل. تضمنت المراجعة المنهجية 24 ورقة نصية، تم تصنيفها إلى ثلاث مجموعات: أولا، الذكاء الاصطناعي في الإدارة، ثانيا: الذكاء الاصطناعي كبديل للقوى العاملة البشرية، ثالثا: مستقبل الذكاء الاصطناعي. وربما أثرت الاختلافات الثقافية على النتائج، لأن الولايات المتحدة ساهمت بأكبر حصة في البحث. كانت

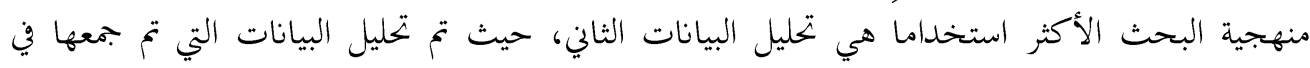
الدراسات السابقة. بناء على نتائج مراجعة الأدبيات المنهجية. ويمكن القول إن المستقبل يؤثر على مدراء

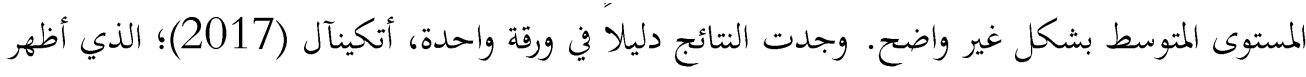


أن إحدى عواقب الذكاء الاصطناعي في إدارة المرافق قد تتمثل في تجاوز المدراء المتوسطين. ومع ذلك، أظهرت أربع أوراق بحثية تأثيرا مستقبليا على المدراء حيث لم يتم تحديد مستواهم الهرمي، مما يعني أنه من غير

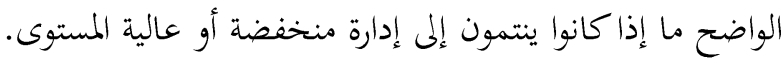

\section{منهج الدراسة:}

منهج الدراسة مبني على الذكاء الاصطناعي ودوره في الأداء المؤسسي. تستخدم الباحثة منهج البحث

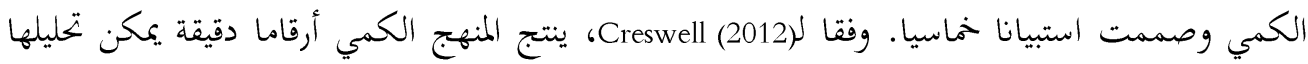
إحصائيا، ويؤدي إلى نتائج لقياس معدل ودرجة أو مستوى الاتجاهات، ويمكنه أيضا تقديم معلومات قيمة

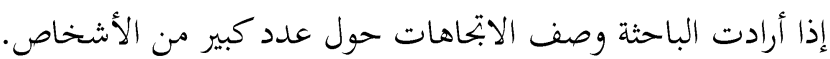

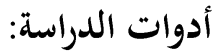

الجمع البيانات بالأسلوب الكمي، في الدراسة الرئيسية قامت الباحثة بإعداد (استبانة) بالاستفادة من الدراسات السابقة: دراسة ياغي (2003)، ربيع زروالي (2019)، دراسة جو يونغ لي (2009).

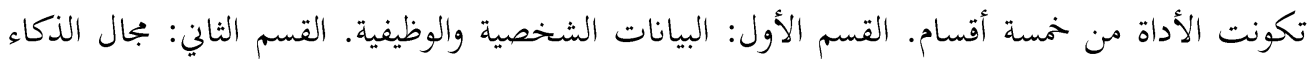
الاصطناعي في الوزارة بما في ذلك الاستشراف والتفكير بالنظم والرؤية المستقبلية والدافعية (القدرة على تحفيز الموظفين) والشراكة في الوزارة. القسم الثالث: تناول بمال دور الاتصال الإداري. والقسم الرابع بمجال اتخاذ القرارات. وأخياً القسم الخامس: تغطية تحسين الأداء المؤسسي. وقامت الباحثة بإجراء تطبيق عملي لاختبار صدق الاتساق وثبات أداة الاستبانة، تلا ذلك دراسة استطلاعية.

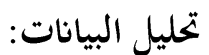
يتم تحليل البيانات الكمية عن طريق الإحصاء الوصفي وتحليل البيانات الأولية والشخصية كالجنس، والعمر، والخبرة، والمؤهل العلمي، والمجال الوظيفي باستخدام SPSS. ثم سيتم استخدام برنامج النمذجة البنائية Smart-PIs (سمارت بي ال اس) لاختبار فرضيات الدراسة والتأكد من مدى ملائمة النموذج.

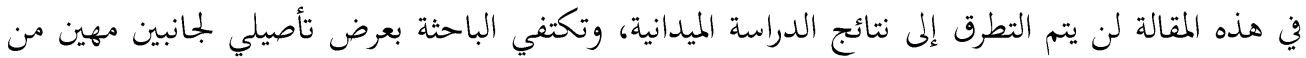
دراستها، يتعلق الأول باتخاذ القرار، والثاني توظيف الذكاء الاصطناعي بوزارة الداخلية. 


\section{المطلب الأول: عملية اتخاذ القرار}

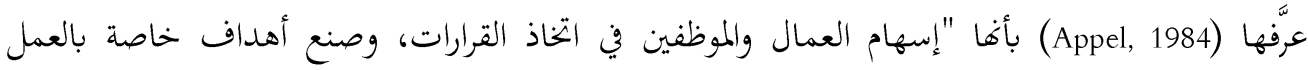
والالتزام بتطبيق تلك القرارات لتحقيق الأهداف". أما (Roberts and Hunt, 1991) فيعرفوفا على أهنا

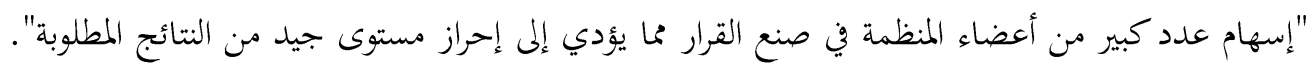

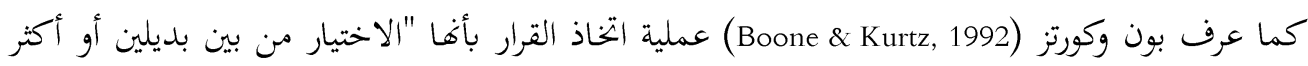

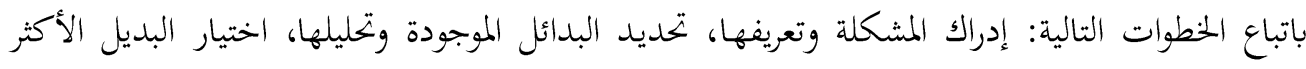
مساهمة في تحقيق أهداف المنظمة وتنفيذه".

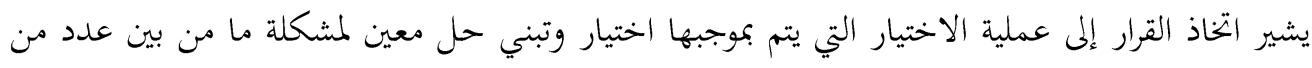

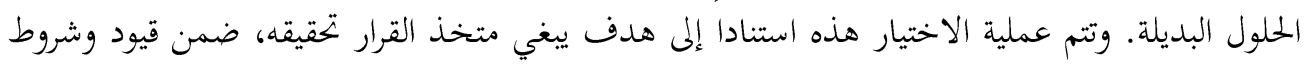

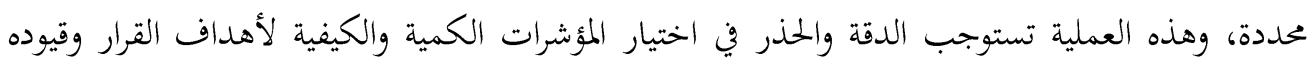

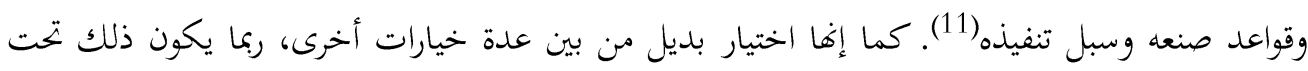
ضغوط وظروف غير مؤكدة (Russell-Jones,2000). تساعد عملية اتخاذ القرار في المسؤوليات الرئيسة التي يتحملها المدير بوصف التخاذ القرارات نشاطاً إدارياً

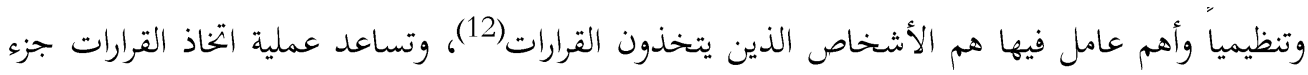

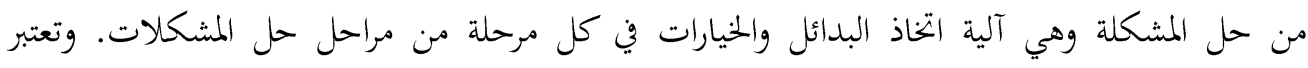

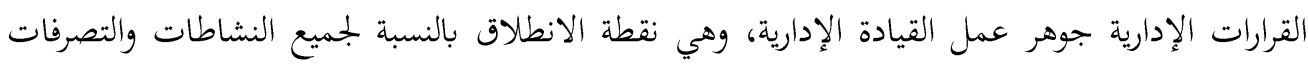

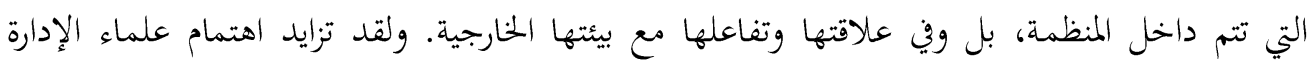
والسلوك التنظيمي بدراسة اتخاذ القرارات. ويؤكد أيوب (1989) أن عملية اتخاذ القرارات هي مظهر أساسي من مظاهر القيادة، لأن المدير يهقق

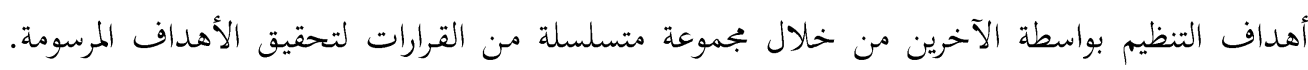

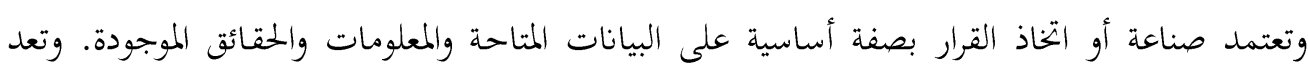

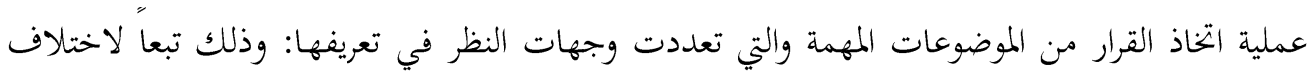

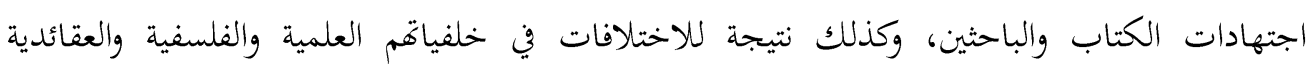
والاجتماعية، وفيما يلي بعض التعريفات.

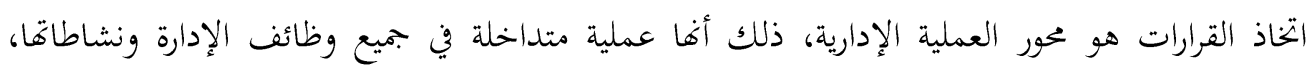

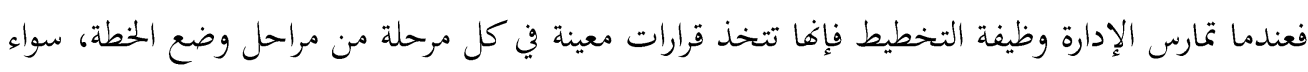


عند وضع الهدف أو رسم السياسات أو إعداد البرامج أو تحديد الموارد الملائمة أو اختيار أفضل الطرق

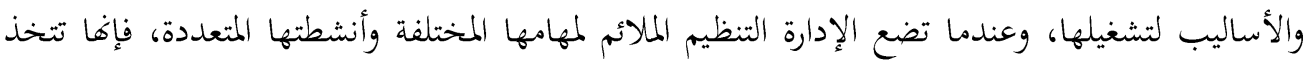
قرارات بشأن الهيكل التنظيمي ونوعه وحجمه وأسس تقسيم الإدارات والأقسام، والأفراد الذين تحتاج إليهم للقيام بالأعمال المختلفة ، ونطاق الإشراف المناسب وخطوط ورئ والسلطة والمسؤولية والاتصال (13). ويشير (ياغي، 2003) إلى أنه من أهم النتائج التي توصلت إليها بتحارب "هوثورن" هي أن الأسلوب

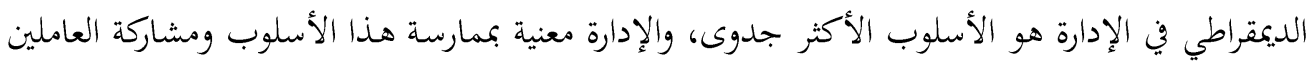
في القرار كنمط قيادي. يعتقد بعض العلماء أن عملية اتخاذ القرارات ينبغي أن تمر بعدة مراحل وخطوات منطقية تمدف في النهاية

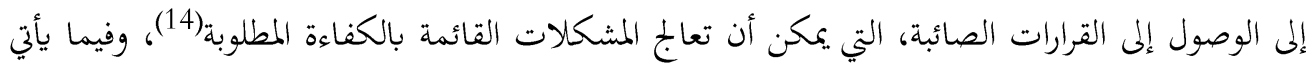
عرض لخطوات اتخاذ القرار التي ينبغي على الفرد إتباعها عند مواجهة مشكلة ما:

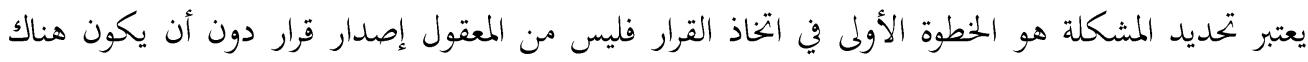
محل أو هدف من وراء إصداره، ويقصد بتحديد المشكلة تشخيصها أي الوقوف على طبيعتها وماهيتها

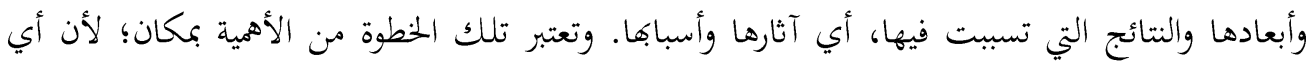

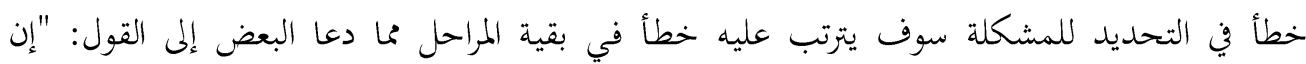
المشكلة المحددة تحديدا واضحا تعتبر نصف محلولة ولذلك فمن المستحسن أن يتم تحديد المشكلة

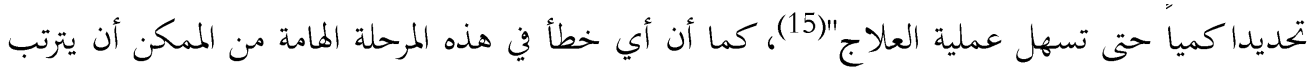
عليه اتخاذ قرارات خاطئة. مرحلة البحث عن الحلول أو القرارات البديلة: وترتكز هذه المرحلة على سلسلة من الافتراضات والتنبؤات التي يقيمها جهاز اتخاذ القرار بقصد التعرف على النتائج المتوقعة، وتعد هذه المرحلة صعبة وشاقة وتتطلب من المدير الاستعانة بآراء الآخرين، ثم دراسة كل حل من الحلول عن طريق تحليله ومعرفة مزاياه وعيوبه،

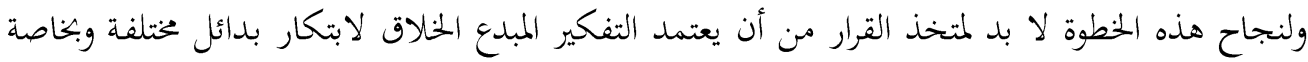

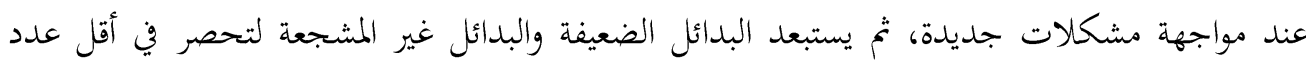

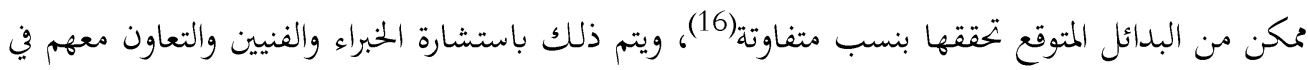
ابتكار بعض البدائل المناسبة(17). في الغالب لا يقوم متخذ القرار بتنفيذ القرار، والذين يقومون بتنفيذه هم أشخاص غير الذين أعدوه ولذلك

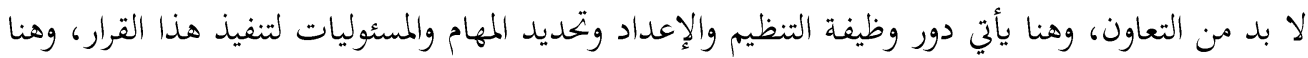


كذلك تدخل عملية التحفيز للموظفين ودفعهم لإنجاز هذا القرار وهذا يتم بواسطة تحفيزهم ماديا ومعنويا

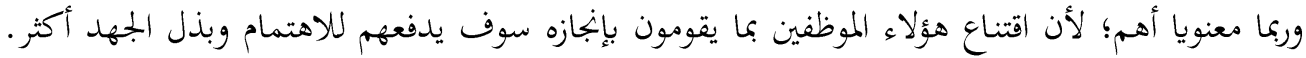

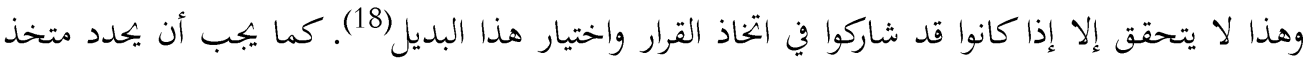
القرار أهدافه بدقة من أجل الاستفادة من آليات التقييم والتحليلات (Simon,2000). إن أية إدارة لا يمكن أن تقف عند حدود تنفيذ القرار لأن متابعة تطبيقه والرقابة في عملية تطبيقه تساعد

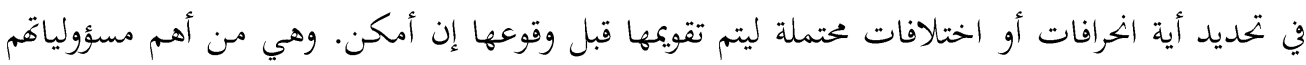

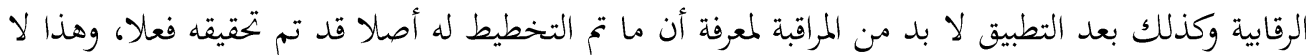
يتم إلا بجمع المعلومات وفحصها وتحليلها لمعرفة النتائج التي تحققت نتيجة لاختيار هذا البديل وتطبيقه وهل كانت النتائج إيجابية أم سلبية(19). تعد الحقائق قواعد ممتازة في التخاذ القرارات، وحين توفرها فإن القرارات المتخذة تصبح ذات جذور قوية ومنطقية. ويقصد بهذا الأسلوب استخدام المدير حكمه الشخصي واعتماده على سرعة البديهة في إدراك العناصر الرئيسية للمواقف والمشكلات التي تعرض له، والتقدير السليم لأبعادها، وفي فحص وتحليل وتهيل وتقييم

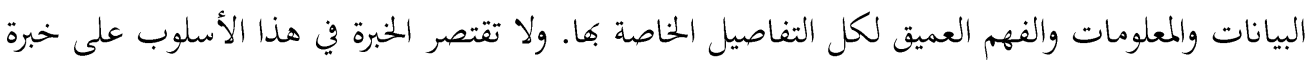
المدير متخذ القرار، ولكن يمكنه التعلم والاستفادة من خبرات المدراء الآخرين ومن زملائه وبتحاربم في حل المشاكل الإدارية واتخاذ القرارات الصائبة. يتولى متخذ القرار نفسه إجراء التجارب آخذا في الاعتبار جميع العوامل الملموسة وغير الملموسة والاحتمالات المرتبطة بالمشكلة محل القرار، حيث يتوصل من خلال هذه التجارب إلى اختيار البديل الأفضل معتمدا في هذا الاختيار على خبرته العملية(20). في هذا الأسلوب يكون اعتماد المدير على البحث ودراسة الآراء والمقترحات التي تقدم إليه حول المشكلة وتحليلها ليتمكن في ضوئها من اختيار البديل الأفضل، وهذا يتطلب العديد من البيانات والإحصائيات

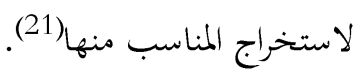
تعتمد عملية اتخاذ القرار الأمثل على قياس الاحتمالات، والاحتمال درجة اعتقاد في حدث ما وقيمة الاحتمال تبدأ من الصفر إذا كان الموقف مستحيل الحدوث وقد تكون إذا كان الموقف متوقع الحدوث بنسبة (100\%) أي مؤكدة(22)، ومن أسباب صعوبة وتعقد هذه المرحلة؛ أن اختيار البديل الأفضل سيتبعه اختيارات لاحقة مما يتطلب من متخذ القرار التأكد من احتمالات المستقبل. وتعتمد الطريقة العلمية في المفاضلة بين البدائل التي يمكن اتخاذها اتحاه مشكلة معينة من خلال المقاييس الرياضية والمنطقية. 
وتشمل بجموعة من النماذج التي تمكن من تحديد الاستراتيجيات التي يوجهها متخذ القرار كأحد المشاركين في مباريات المنافسة وكيفية الوصول إلى الاستراتيجية الأفضل.

\section{المطلب الثاني: توظيف الذكاء الاصطناعي في وزارة الداخلية في دولة الإمارات العربية المتحدة}

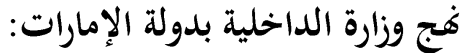

اتبعت الوزارة منهجا جديدا يرتكز على أسس علمية ومنهجية لتطوير الإمكانيات البشرية والمادية لوزارة الداخلية لمواكبة النهضة الشاملة التي تشهدها الدولة في مختلف الجمالات.لا شك أننا أمام تحديات كثيرة وأن

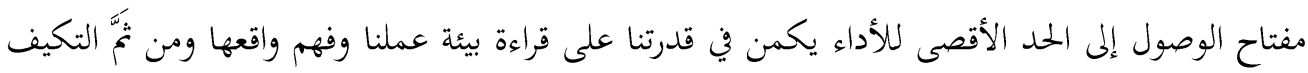
معها، وينبغي أن نستمر في استكشاف طرق جديدة لترسيخ الحوار الواعي مع مختلف فئات الجمتمع ولتوفير الطمأنينة لدى أفراده مع الاستمرار في تلبية حاجاهم المتغيرة بالشكل المناسب.

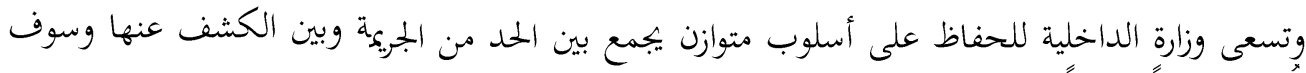

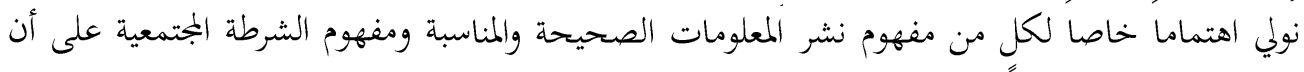
نتعاون في هذا الصدد مع كافة السلطات والهيئات والجهات المعنية. لذلك فئل إنَّ الاستراتيجية الأمنية لوزارة الداخلية تتطلب استشراف المستقبل والنطلع إليه، وينبغي حضور الإرادة والاستعداد للتجاوب بمرونة

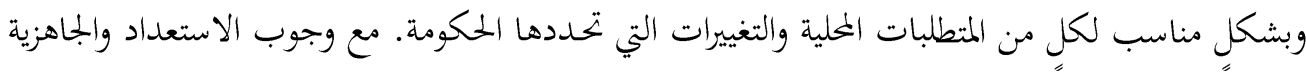
للتغيرات ومواكبة التطورات العالمية. حققت وزارة الداخلية الكثير من الإنجازات والنجاحات من خلال مشروعات التوسعة والتجديد القادرة

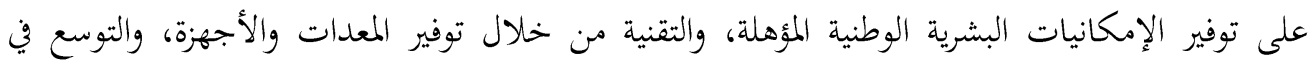
تطوير الخدمات المقدمة للجمهور وفق خطط استراتيجية أمنية فعالة تستوعب متطلبات الحاضر والمستقبل قائمة على روح التعاون والمبادرة وتفعيل الشراكة الجمتمعية بين الأجهزة الأمنية والمجتمع أفراد وهيئات ومنظمات لتحقيق مفهوم المسؤولية المشتركة في توفير المزيد من الأمن والاستقرار في المجتمع. وتعد وزارة

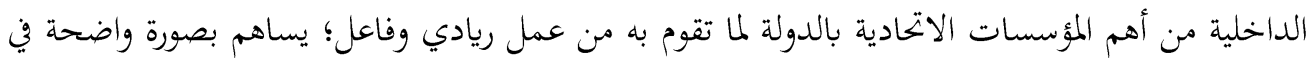
دعم الأمن والاستقرار فيها وهي تنطلق من غاية سامية هي إيجاد مجتمع أكثر أمانا والحفاظ على النظام

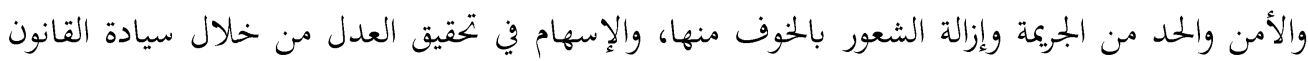
وحفظ هيبة الدولة. 
وقامت الوزارة بتبني أسلوب إداري متناسق ومتناغم كما استطاعت تلبية هتطلبات جديدة، لإرساء وترسيخ

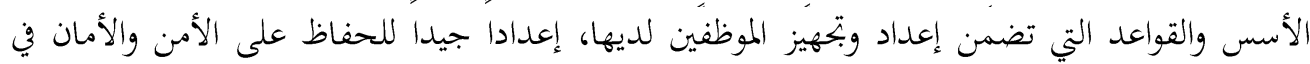

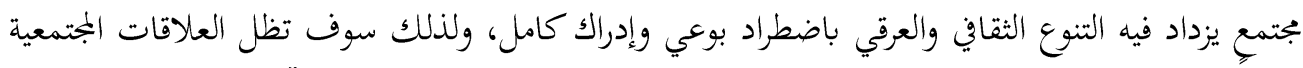

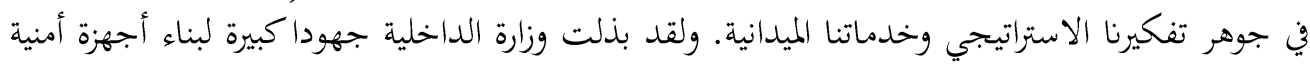

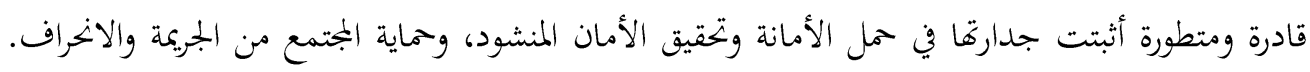
مما جعل دولة الإمارات دولة عصرية قبلة المستثمرين.

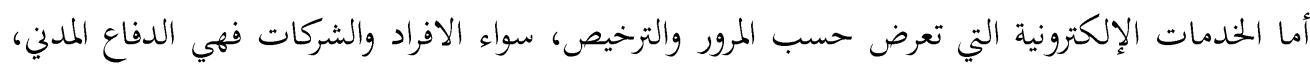

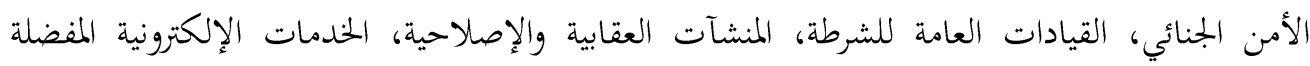

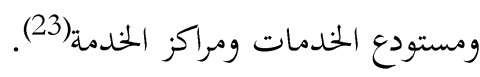

\section{الذكاء الاصطناعي في القطاعات الحيوية للدولة:}

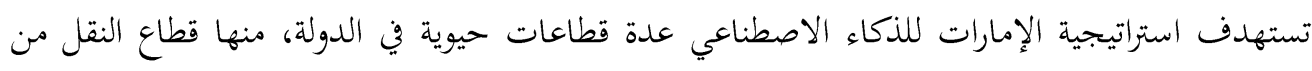

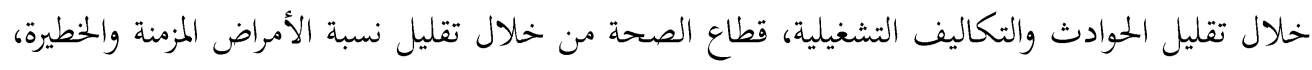

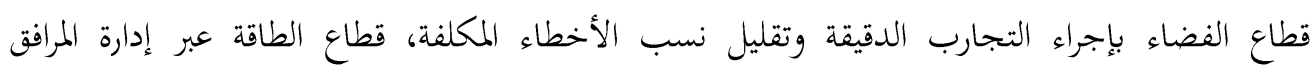

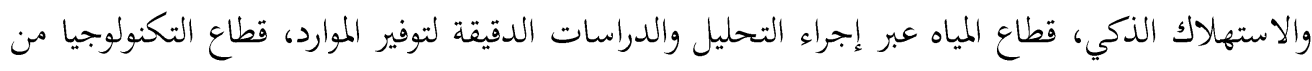

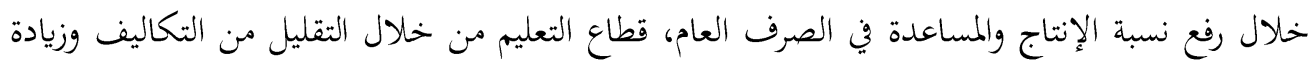

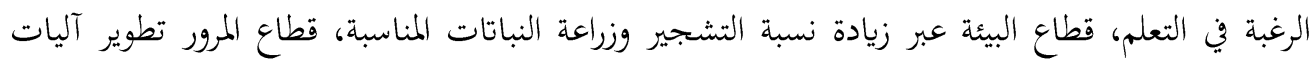
وقائية كالتنبؤ بالحوادث والازدحام المروري، ووضع سياسات مرورية أكثر فاعلية.

\section{مؤشرات جاهزية الحكومة الالكترونية في دولة الإمارات:}

يتكون مؤشر جاهزية الحكومة الالكترونية كالآتي:

الأول: يتضمن منهجية قياس مؤشر الجاهزية الإلكترونية للنهوض بمستوى أداء الإدارات العامة الحكومية

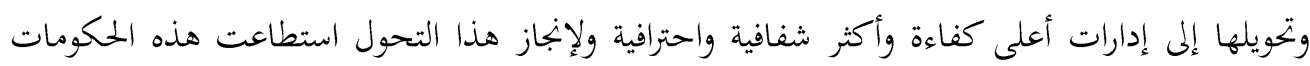

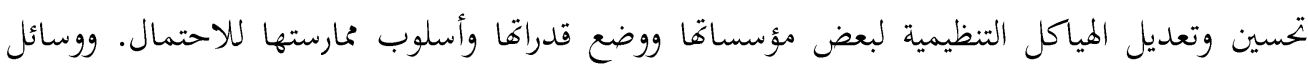

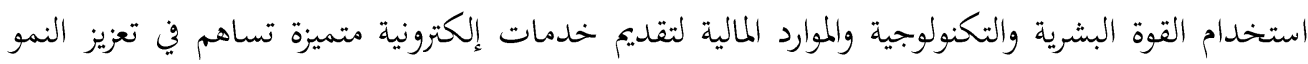
الاقتصادي والاجتماعي للمجتمع وتلقى قبولا ورضى من متلقى هذه المخدمات. 
الثاني: تعريف المؤشر الذي صدر في فبراير 2008م أحدث إصدار لمؤشر جاهزية الحكومة الإلكترونية Government Index لهذا المؤشر في عام 2003م. ويقيس المؤشر مدى التحول نحو إصلاح وتنمية القطاع العام والمؤسسات

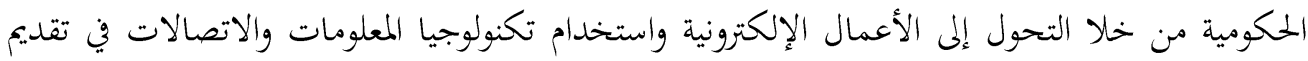

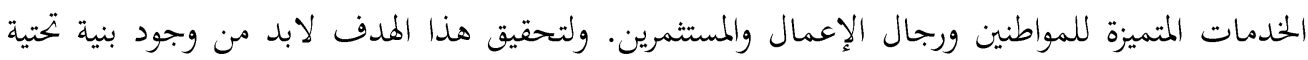
قوية من تكنولوجيا المعلومات والاتصالات. كما يحفز المؤشر دول العالم إلى تغيير ثقافة تقديم الحندمات الحكومية الإلكترونية وتشجيع العاملين بالقطاع الحكومي لاستخدام وسائل تقنية حديثة لسرعة وسهولة لحئل إنجاز الإعمال. الثالث: مكونات المؤشر والذي يتضمن المؤشر المركب لجاهزية الحكومة الإلكترونية يشمل تقييم الوضع في (192) دولة على مستوى العالم من خلال مسح أجرته الأمم المتحدة لقياس مد استفادة المواطنين

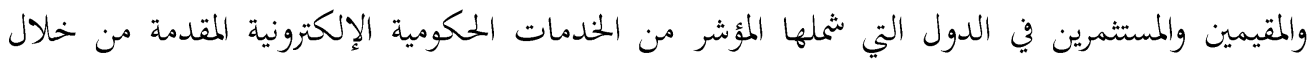

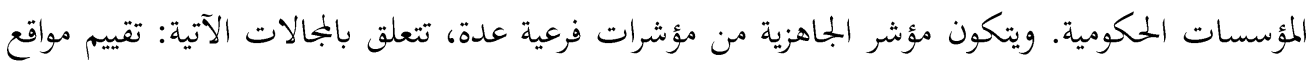
الانترنت الحكومية الخدمية. البنية الأساسية للاتصالات والمعلومات. الموارد البشرية (رأس المال البشري). الرابع: مؤشر تقييم مواقع الإنترنت: الذي يقيم إجمالي قدرة الحكومات على تقديم خدمات إلكترونية من

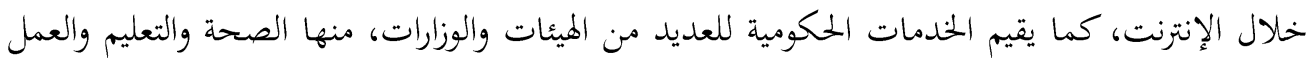

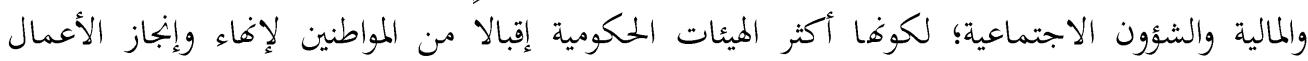
والخدمات.

الخامس: مؤشر البنية التحتية للاتصالات والمعلومات: وهو مؤشر مركب من (5) مؤشرات فرعية يعكس

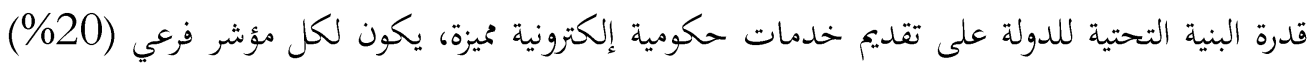
من إجمالي نقاط مؤشر البنية التحتية للاتصالات والمعلومات. وهذه المؤشرات هي: أ. مؤشر عدد مستخدمي الإنترنت (لكل 100 شخص). ب. مؤشر عدد الحاسبات (لكل 100 شخص). ت. مؤشر عدد الخطوط التليفونية الأرضية (لكل 100 شخص). ث. مؤشر عدد خطوط المحمول (لكل 100 شخص). ج. مؤشر عدد خطوط الإنترنت السريع (لكل 100 شخص). 
السادس: مؤشر الموارد البشرية يتكون من: معدل محو الأمية (ثلثي النقاط). التأجيل في المراحل الابتدائية والإعدادية والثانوية (ثلث النقاط). وتستقى البيانات من اليونسكو وتقارير التنمية البشرية للأمم المتحدة.

ناقشت هذه المقالة، عملية اتخاذ القرار، وتوظيف الذكاء الاصطناعي في دولة الإمارات العربية المتحدة. ومن خلال المنهج الوصفي التحليلي تم عرض الموضوع في مطلبين، وقد بينت المناقشات أنَّ استراتيجية الإمارات للذكاء الاصطناعي تستهدف عدة قطاعات حيوية في الدولة، منها قطع النقل لتقليل الحوادث والتكاليف التشغيلية. قطاع الصحة لتقليل الأمراض المزمنة والخطيرة. قطاع الطاقة لتحقيق الاستهلاك الذكي. قطاع المياه لتوفير الموارد. قطاع التكنولوجيا من خلال رفع نسبة الإنتاج. قطاع التعليم لتقليل التكاليف وزيادة التعلم. قطاع البيئة لزيادة التشجير وزراعة النباتات. قطاع المرور للتنبؤ بالحوادث والازدحام المروري.

1. الحسيني، أسامة. (2002). لغة لوجو. الرياض، مكتبة بن سينا للنشر والثوزيع، الطبعة الاولى.

2.

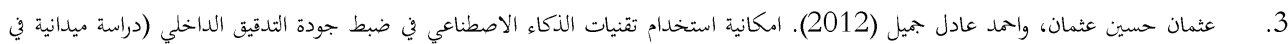

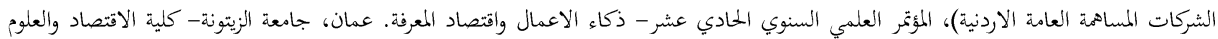

الادارية.

عبد الجبيد، قتيبة مازن. (2009). استخدام الذكاء الصناعي في تطبيقات الهندسة الكهربائية: دراسة ومقارنة. دراسة ماجستير في نظم المعلومات الإدارية،

الأكاديمية العربية في الدنمارك.

قمورة، سامية شهبي. (2018). الذكاء الاصطناعي بين الواقع والمأمول: دراسة تقنية ميدانية. ورقة بحثية مقدمة إلى الملتقى الدولي "الذكاء الاصطناعي:

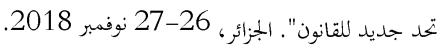

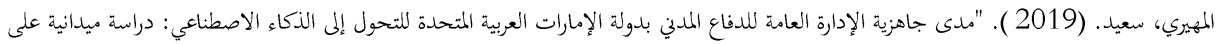

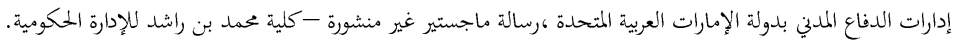

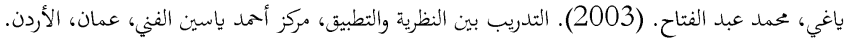

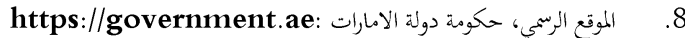

9. الموقع الرسمي للإمارات اليوم (2018)،الثقرير الصادر عن مركز دبي التكنولوجي لريادة الاعمال (ديتك) التابع لسلطة واحة دبي للسيليكون، المنشور في

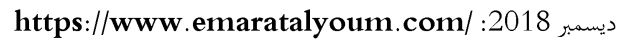

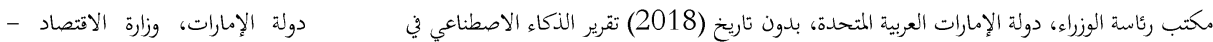

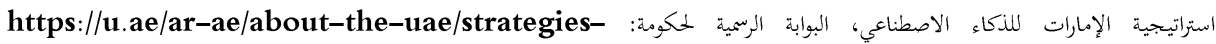

initiatives-and-awards/federal-governments-strategies-and-plans/uae-strategy-forartificial-intelligence

https://u.ae/ar-ae/about-the-uae/strategies- استراتيجية الإمارات للذكاء الاصطناعي - البوابة الرسمية لحكومة:

initiatives-and-awards/federal-governments-strategies-and-plans/uae-strategy-forartificial-intelligence 


$$
\begin{aligned}
& \text { الهوامش: } \\
& \text { (1) خضر، سعاد، } 2000 . \\
& \text { (2) الحسيني، } 2002 . \\
& \text { (3) ياغي، (2003. } \\
& \text { (4) إيلي باريسر، } 2012 . \\
& \text { (5) عرنوس، } 2007 .
\end{aligned}
$$

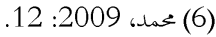

$$
\begin{aligned}
& \text { (7) جابر، } 2003 . \\
& \text { (8) مطاي، } 2012 . \\
& \text { (9) ملوخية، } 2007 . \\
& \text { (10) ملوخية، } 2007 . \\
& \text { (11) شمس الدين، } 2005 . \\
& \text { (12) موسى، } 2010 \text { (13 الدين، } \\
& \text { (13) القريوي، (12) موسى، } 2004 . \\
& \text { (14) عبوي، } 2010 . \\
& \text { (15) تعلب، } 2011 . \\
& \text { (16) موسى، } 2010 . \\
& \text { (17) عبوي، } 2010 . \\
& \text { (18) حسين والساعد، } 2001 . \\
& \text { (19) حسين والساعد، } 2001 . \\
& 2003 \text {. } 203 \text { (20) } \\
& \text { (21) موسى، } 2010 \text { (20) } \\
& \text { (22) موسى، موسى، } 2010 . \\
& \text { (23) موقع وزارة الداخلية، } 2020 .
\end{aligned}
$$

